\title{
Ethanol Lock Therapy as additional treatment of catheter-related bloodstream infections in pediatric patients receiving home parenteral nutrition
}

\author{
Merel N. van Kassel ${ }^{1}$, Dasja Pajkrt ${ }^{2}$, Jim C. H. Wilde ${ }^{3}$, Cora F. Jonkers ${ }^{1}$, Merit M. Tabbers ${ }^{1 *}$ \\ ${ }^{1}$ Home Parenteral Nutrition Team, Emma’s Children’s Hospital Academic Medical Centre, Amsterdam, The Netherlands \\ ${ }^{2}$ Department of Pediatric Infectious Diseases, Emma's Children's Hospital Academic Medical Centre, Amsterdam, The Netherlands \\ ${ }^{3}$ Department of Pediatric Surgery, Emma's Children's Hospital Academic Medical Centre, Amsterdam, The Netherlands \\ Email: ${ }^{*}$ m.m.tabbers@amc.nl
}

Received 20 November 2013; revised 7 December 2013; accepted 15 December 2013

Copyright (C) 2013 Merel N. van Kassel et al. This is an open access article distributed under the Creative Commons Attribution License, which permits unrestricted use, distribution, and reproduction in any medium, provided the original work is properly cited.

\begin{abstract}
Background: Catheter related bloodstream infections (CRIs) are a major cause of morbidity, mortality and prolonged hospitalization in pediatric patients with home parenteral nutrition (HPN). In recent years, new prophylactic and treatment options have been evaluated to reduce the incidence of CRIs and infection related catheter removals. In children, two retrospective studies have shown the efficacy of ethanol lock treatment (ELT) in reducing the incidence of CRIs in pediatric oncology patients. However, prospective data are missing. Our aim of the present study was to evaluate prospectively the effect of ELT on the cure and recurrence rate of CRIs, duration of hospitalization and antibiotic use in HPN pediatric patients. Methods: HPN children older than three months with a clinical suspicion of a CRI were included and randomised to ELT or standard flushing treatment (heparin or taurolidine). The primary outcomes were persistence and recurrence of the bacteraemia and Central Venous Access Device (CVAD) removal. Secondary outcomes included duration of antibiotic use, duration of hospitalization and side effects. Results: Data from eight HPN children experiencing 14 suspected CRI were analysed. Seven patients had proven CRIs by positive blood cultures. Four were treated with ELT and three with their standard flushing treatment. One CRI persisted in the control group. In one single patient, bacteraemia recurred three times within 24 weeks. This patient had been enrolled in both study arms. A CVAD removal was needed in one patient because of occlusion. Conclusions: This small study showed no additional
\end{abstract}

"Corresponding author. efficacy of the use of ELT on cure rate or recurrences of CRIs compared to standard therapy in HPN treated children. There were also no differences in duration of hospitalization, the use of antibiotics or CVAD removal between both groups.

Keywords: Home Parenteral Nutrition; Ethanol Lock Treatment; Catheter Related Bloodstream Infections

\section{INTRODUCTION}

For children depending on Home Parenteral Nutrition (HPN), catheter related bloodstream infections (CRIs) are a major source of morbidity, mortality and prolonged hospitalization [1-8]. A rate from 5.7 catheter-related infections per 1000 catheter days has been reported in pediatric patients with intestinal failure as compared to 11.2 catheter-related infections per 1000 catheter days in HPN pediatric patients with short bowel syndrome (a group with high risk for CRIs) [4,9]. Due to limited venous accesses in the pediatric population, Central Venous Access Device (CVAD) replacements are limited [10]. Most CRIs occur after contamination of the hub during manipulation or infusion of contaminated medication or fluids, with subsequent migration of microorganisms to the intra-luminal surface and formation of a microbial biofilm [5].

In order to reduce the incidence of the CRIs and catheter removals, new methods of prophylaxis and treatment have been evaluated in recent years. In vitro studies have shown that ethanol has a broad-spectrum antimicrobial activity against Candida and both Grampositive and negative bacteria, without an adverse effect on the integrity of the CVADs and with a decreased risk of developing drug resistance [11-15]. Furthermore, 
ethanol has the advantage of being inexpensive and universally available [16].

A descriptive retrospective study showed a beneficial effect of Ethanol Lock Therapy (ELT) in reducing the incidence of CRIs in adult HPN patients [17]. In children, only retrospective studies have been performed to evaluate the preventive effect of ELT on CRIs. A small retrospective study in pediatric patients with intestinal failure receiving long-term HPN showed that the mean CRI rate per 1000 catheter days significantly decreased from 8.0 before ELT to 1.3 after ELT $(\mathrm{P}<0.01)$ [9].

Two retrospective studies have reported the valuable effect of ELT as an additional therapy to systemic antibiotics to CRIs in children with long-term CVADs with diverse underlying disorders $[6,18]$. One of these studies even showed $67 \%$ of 28 children treated with ELT and systemic antimicrobial therapy cleared the infection with no relapse within 4 weeks compared to $47 \%$ in those who were treated with systemic antimicrobial therapy alone [18]. However, prospective pediatric studies concerning the additional therapeutic effect of ELT on CRIs in patients with are lacking. For this reason, our aim was to evaluate prospectively the effect of ELT in combination with antibiotics as treatment for CRIs in pediatric patients on HPN compared to the standard flushing treatment with antibiotics.

\section{MATERIALS AND METHODS}

The patient database of the Emma Children's Hospital/ Academic Medical Centre, Amsterdam, the Netherlands was used. All children on HPN and a CVAD, which was used at least twice a week for more than a month and with a suspicion of a catheter related bloodstream infection, were included from October 2009 to July 2011. Eligible patients were randomised to be treated with either ELT or standard flushing therapy. Included patients with a clinical suspicion of a CRI were being treated with systemic empirical antibiotic treatment after blood sampling for microbial blood cultures. They also received ELT or standard flushing therapy during the whole period of antibiotic use. After this antibiotic period they returned to their standard flushing treatment.

At the start of our study the standard flushing treatment consisted of $3 \mathrm{ml}$ heparin $100 \mathrm{U} / \mathrm{ml}$. In 2010 the standard flushing treatment was altered into a combination of a $3 \mathrm{ml}$ taurolidine $2 \%$ solution. Taurolidine is an antiseptic agent derivative from the naturally occurring aminosulphonic acid taurinamide and formaldehyde, with a broad spectrum of antimicrobial activity against Gram-positive bacteria, Gram-negative bacteria and fungi [18].

The local Medical Ethical Committee approved the study and each patient and/or caregivers provided written informed consent before inclusion.
In this randomised open label study the effect of ELT on the persistence of CRIs, recurrence of CRIs, duration of hospitalization and duration of antibiotic use in HPN pediatric patients older than three months of age compared to the standard treatment (heparin or taurolidine solution) were monitored.

\subsection{Inclusion and Exclusion Criteria}

We included: 1) children with a CVAD; 2) active use of the CVAD for HPN (minimal use of twice a week for more than a month); 3) suspicion of a catheter related bloodstream infection for which, according to the treating physician, antimicrobial therapy is indicated.

We excluded: 1) patients allergic to ethanol or alcohol based on the medical history of the patient; 2) patients in need of vasoactive medication or mechanical ventilation; 3) patients with Staphylococcus Aureus or Candida CRI and in the event a CVAD could be removed with alternative CVAD replacement options; 4) patients depending on a catheter for 24 hours or with continuous use of the CVAD

All the included patients with a suspicion of a CRI were treated with systemic broad-spectrum antimicrobial therapy. After susceptibility assessment of the causative microorganism cultured from blood, antibiotic treatment was adjusted accordingly.

\subsection{Main Study Parameters}

The primary outcomes included persistence of the bacteraemia, defined as a positive blood culture seven days after start of ELT. Secondly, the recurrence of the bacteraemia, defined by a positive blood culture with the same or other microorganism within 24 weeks and finally removal of CVAD. Secondary measurements included duration of antibiotics use, duration of hospitalization due to CRIs and possible side effects of ELT.

\subsection{Procedures}

Prior to initiation of systemic antimicrobial treatment and instillation of ELT or standard flushing therapy, paired blood cultures were obtained from the CVAD and peripheral blood. CRI is proven with positive blood cultures of the CVAD. After seven days a new blood culture from the CVAD and peripheral blood were taken. Additional blood cultures were obtained in case a recurrent or relapsing CRI was suspected. During ELT the following symptoms were recorded: tiredness, headaches, dizziness, nausea and light-headedness.

Patient characteristics, duration of hospitalization and antibiotic use were obtained from the medical files and put into a database.

The standard lock consists of $3 \mathrm{ml}$ heparin $100 \mathrm{U} / \mathrm{ml}$. The ethanol locks were prepared using the following 
procedure: $2.0 \mathrm{ml}$ 96\% Ethanol was withdrawn with filter needle into a $10 \mathrm{ml}$ syringe. Subsequently, $1 \mathrm{ml} \mathrm{NaCl}$ $0.9 \%$ was added to the withdrawn $96 \%$ ethanol syringe to make a final concentration $67 \%$ alcohol. Patients received $67 \%$ ethanol instilled into the lumen of the catheter.

All patients in the ELT group received the ELT for a minimum duration three to six hours per 24 hours. After the required dwell time the ethanol lock was withdrawn, discarded and followed by a 10 cc $\mathrm{NaCl} 0.9 \%$ flush. This procedure was repeated for the entire term the patient was treated with antibiotics. After the ELT or standard lock therapy in combination with antibiotic treatment patients continued the use of their standard preventive therapy (heparin or taurolidine).

\section{RESULTS}

The median rate of CRIs proved by a positive blood culture combining ELT and standard lock treatment during our study period was 1.6 infections per 1000 catheter days (range 0 to 4.82 infections per 1000 catheter days).

In Table 1 is shown that the median age was 3.5 years (SD = 2.86), three years for the ELT group and four years for the control lock group. Underlying diagnoses included intestinal pseudo-obstruction syndrome, microvillus inclusion disease, short bowel and intestinal motility disorder. Most of the CVAD were single lumen Broviac catheters.

In Table 2 is shown that during the study period, eight children experienced a suspected CRI on 14 occasions. A

Table 1. Patient characteristics.

\begin{tabular}{|c|c|}
\hline Characteristic & n (\%) \\
\hline \multirow[t]{2}{*}{$\begin{array}{l}\text { Age at the moment of } \mathrm{CRI} ; \mathrm{n}=14^{*} ; \\
\text { range (mean } \pm \mathrm{SD} ; \text { median) }\end{array}$} & $18 \mathrm{mo}$ to $12 \mathrm{y}$ \\
\hline & $(4.5 \pm 2.9 y ; 3.5 y)$ \\
\hline \multicolumn{2}{|l|}{ Gender $(\mathrm{n}=8)$} \\
\hline Male & 4/8 (50) \\
\hline Female & $4 / 8(50)$ \\
\hline \multicolumn{2}{|l|}{ Primary Diagnosis $(\mathrm{n}=8)$} \\
\hline Intestinal pseudo-obstruction & $4(50)$ \\
\hline Microvillus Inclusion Disease & $2(25)$ \\
\hline Short Bowel & $1(12.5)$ \\
\hline Intestinal motility disorder & $1(12.5)$ \\
\hline \multicolumn{2}{|l|}{ Catheter Type $(n=14)$} \\
\hline Single lumen Broviac & $13(93)$ \\
\hline Porth-a-cath & $1(7)$ \\
\hline
\end{tabular}

\footnotetext{
*The 8 patients in this analysis experienced 14 times suspected CRIs.
}

Table 2. Overview of endpoints.

\begin{tabular}{ccc}
\hline Parameter & Ethanol $(\mathbf{n}=\mathbf{7})$ & Standard $(\mathbf{n}=\mathbf{7})$ \\
\hline Age at Moment CRI & 3 to 9 years & 1.5 to 12 years \\
Positive CRI & $4.3 \pm 2.2 \mathrm{y} ; 3 \mathrm{y})$ & $(4.6 \pm 3.6$ y; 4 y) \\
Persistence of CRI & 5 & 3 \\
Recurrence of CRI & 1 & 1 \\
CVAD Removal & 1 & 1 \\
Symptomatic \\
Venous Thrombosis \\
$\begin{array}{c}\text { Duration } \\
\text { Hospitalization (Days) } \\
\text { Duration Antimicrobial } \\
\text { Use (Days) }\end{array}$ & 4 days & 0 \\
Positive Blood Culture & 14 days & 3 days \\
Negative Blood Culture & 7 days & 18.5 days \\
\hline
\end{tabular}

"Because of pre-admission occlusion.

CRI was proven five times in the ELT group by positive blood cultures as compared to three proven CRIs in the standard lock group. Among the total of eight HPN patients with a proven CRI, six patients were treated once, one patient was treated twice (once in the ELT arm and once in the control lock arm) and one received the therapy six times (four times in the ELT arm, twice in the control arm).

Table 3 shows the primary and secondary outcomes. Among the fourteen suspected CRIs, seven were proved, including six in the CVAD. Of all the proven infections, four were treated in the ELT group and three with their standard flushing regimen (heparin or taurolidine solution). Of all the proven line infections, one persisted after seven days in the heparin group. One case (patient 5) had initially no proved central or peripheral infection, however after seven days a peripheral infection with coagulase-negative staphylococci had been found. In one single patient, bacteraemia recurred three times within 24 weeks. First time with pathogen Klebsiella (case 3), eleven weeks later with pathogen Pseudomonas (case 6) and five weeks later with the same pathogen Pseudomonas again (case 8). In case 3 the patient was treated with ELT, and in case 6 the patient was treated with heparin solution.

Patient 12 had a CRI caused by S. aureus, in whom the CVAD could not be removed because of lack of alternative CVAD replacement localisations. A CVAD removal occurred in one patient (patient 13) because of pre-admission occlusion.

The caregivers of patient 14 did not want to switch to taurolidine as new standard flushing treatment, so for this reason patient 14 is still using heparin as standard flushing treatment. 
Table 3. List with duration hospitalization, antibiotics used and bacteraemia results.

\begin{tabular}{|c|c|c|c|c|c|c|}
\hline Pt & $\begin{array}{c}\text { Duration } \\
\text { Hosp. }\end{array}$ & $\begin{array}{l}\text { Treatment Antibiotics } \\
\text { and Duration }\end{array}$ & $\begin{array}{l}\text { Positive Blood Culture } \\
\text { on Admission Day } 0\end{array}$ & $\begin{array}{c}\text { Positive Blood Culture } \\
\text { after } 7 \text { Days }\end{array}$ & $\begin{array}{l}\text { Recurrence Bacteraemia } \\
\text { within } 24 \text { Weeks }\end{array}$ & $\begin{array}{l}\text { CVAD } \\
\text { Removal }\end{array}$ \\
\hline $1 \mathrm{E}$ & 9 days & V/G, 7 days & $\mathrm{N}$ & $\mathrm{N}$ & $\mathrm{N}$ & $\mathrm{N}$ \\
\hline $2 \mathrm{H}$ & 3 days & V/G, 3 days & $\mathrm{N}$ & $\mathrm{N}$ & $\mathrm{N}$ & $\mathrm{N}$ \\
\hline $3 \mathrm{E}$ & 2 days & Cfx, 14 days & $\begin{array}{c}\text { Y: C/P C: Klebsiella } \\
\text { P: Co-neg. staphylococci }\end{array}$ & $\mathrm{N}$ & $\mathrm{Y}$ & $\mathrm{N}$ \\
\hline $4 \mathrm{H}$ & 1 day & V/G, 7 days & $\mathrm{N}$ & $\mathrm{N}$ & $\mathrm{N}$ & $\mathrm{N}$ \\
\hline $5 \mathrm{E}$ & 4 days & V/G, 7 days & $\mathrm{N}$ & $\begin{array}{l}\text { Y: P P: Co-neg. } \\
\text { staphylococci }\end{array}$ & $\mathrm{N}$ & $\mathrm{N}$ \\
\hline $6 \mathrm{H}$ & 1 day & Cfz, 11 days & Y: C C: Pseudomonas & Y: C C: Pseudomonas & $\mathrm{Y}$ & $\mathrm{N}$ \\
\hline $7 \mathrm{~T}$ & 3 days & Cfz/Teico, 3 days & $\mathrm{N}$ & $\mathrm{N}$ & $\mathrm{N}$ & $\mathrm{N}$ \\
\hline $8 \mathrm{E}$ & 3 days & Cfz/Tobra, 14 days & Y: C C: Pseudomonas & $\mathrm{N}$ & $\mathrm{N}$ & $\mathrm{N}$ \\
\hline $9 \mathrm{E}$ & 0 days & $\begin{array}{l}\text { A/Cfz, } 3 \text { days; } \\
\text { Fluco } 14 \text { days }\end{array}$ & Y: C C: Pseudomonas & $\mathrm{N}$ & $\mathrm{N}$ & $\mathrm{N}$ \\
\hline $10 \mathrm{E}$ & 4 days & V, 7 days & Y: P P: Micrococcus Specie & $\mathrm{N}$ & $\mathrm{N}$ & $\mathrm{N}$ \\
\hline $11 \mathrm{~T}$ & 3 days & V/G, 3 days; V, 4 days & $\mathrm{N}$ & $\mathrm{N}$ & $\mathrm{N}$ & $\mathrm{N}$ \\
\hline $12 \mathrm{~T}$ & 0 days & $\begin{array}{l}\text { V/G, } 3 \text { days; } \\
\text { Fluco, } 20 \text { days }\end{array}$ & Y: C/P C/P: Staph. aureus & $\mathrm{N}$ & $\mathrm{N}$ & $\mathrm{N}^{*}$ \\
\hline $13 \mathrm{E}$ & 17 days & V/G, 3 days; V, 11 days & $\mathrm{N}$ & $\mathrm{N}$ & $\mathrm{N}$ & $\mathrm{Y}$ \\
\hline $14 \mathrm{H}$ & 11 days & Meropenem, N.A & Y: C C: Citrobacter & $\mathrm{N}$ & $\mathrm{N}$ & $\mathrm{N}$ \\
\hline
\end{tabular}

E, Ethanol-lock; H, Heparin; T, Taurolidine; V, Vancomycine; G, Gentamycine; Cfx, Ceftriaxon; Cfz, Ceftazidim; A, Augmentin; Teico, Teicoplanine; Tobra, Tobramycine; Flu, Flucloxacilline; Y, Yes; N, No; C, Central; P, Peripheral; Hosp, Hospitalization; Co-neg, Coagulase-negative; Staph, Staphylococcus. * Although an infection with Staphylococcus aureus normally means a CVAD removal, we have treated this patient successfully with antibiotics: see text for explanation.

HPN children in the ELT arm with a clinical suspicion of a CRI with negative blood cultures were treated with empiric broad-spectrum antimicrobial therapy (vancomycin and gentamicin) for a median duration of seven days (range 7 - 14 days), while patients in the standard arm were treated for a median duration of five days (range 3 - 7 days). In the ELT group, proven CRIs were treated for a median duration of 14 days (range 7 - 17 days) compared to 18.5 days (range 14 - 23 days) in the standard arm.

The duration of hospitalization was shorter in the heparin group. Median duration of hospitalization in the ELT group was four days (range 0 - 17 days), while in the control group the median duration three days (range 0 - 11 days).

Possible side effects of ELT were noticed in two patients. A nine year old stated he had two days of headache with cold chills. A five year old stated he had stomach pain and nausea.

None of our patients died.

\section{DISCUSSION}

In this study ELT did not show additional efficacy as compared with standard therapy. We included 14 patients with a clinical suspicion of a CRI, seven of them proved by positive blood cultures. No major differences were observed in the reduction of hospitalization duration, duration systemic antimicrobial treatment or CVAD removals. Furthermore, no differences were found between the ELT and control group in persistence or recurrences of CRI. This lack of differences might be explained by the small sample size and the fact that other confounding factors (such as the specific underlying disease of the HPN patients and the causative microorganism) are more important factors in determining the duration of hospital stay and antibiotic use. In addition, the length of hospital stay is depending on the clinical condition of child and the ability of the parents/caregivers to treat the child at home with antibiotics and (ELT or standard) flush/lock therapy.

During the study period the median rate of CRI proven by positive blood culture was 1.56 infections per 1000 catheter days. This is lower as reported previously and might be explained by the alteration of heparin to the broad-spectrum antiseptic agent taurolidine as a component of the standard lock therapy resulted in important decrease in CRI in the standard lock therapy group $[4,10]$. Limitation of this study was the switch of heparin to taurolidine during the study; however this change was made according to the latest scientific insights which reported a beneficial effect of taurolidine in the incidence 
of CRI in pediatric patients. A small prospective study showed a beneficial effect of taurolidine in the occurrence of CRI in adult HPN patients (from $71 \%$ in the taurolidine group as compared to $6 \%$ in the heparin group) [19]. A small retrospective study demonstrated a beneficial effect of taurolidine lock in reducing the incidence of CRIs in pediatric HPN patients [20-22]. Based on these results, we suggest a multicenter randomised trial evaluating the efficacy and safety of taurolidine in pediatric patients as preventive or therapeutic treatment of CRIs in HPN children as compared to ELT.

\section{CONCLUSION}

Our results demonstrate that ELT was not more effective in reducing persistence and recurrence of CRIs as compared to standard treatment, but larger prospective trials are needed to really determine the efficacy of ELT as additional treatment of CRIs in children with HPN.

\section{REFERENCES}

[1] Wales, P.W., Kosar, C., Carricato, M., De Silva, N., Lang, K. and Avitzur, Y. (2011) Ethanol lock therapy to reduce the incidence of catheter-related bloodstream infections in home parenteral nutrition patients with intestinal failure: Preliminary experience. Journal of Pediatric Surgery, 46, 951-956. http://dx.doi.org/10.1016/j.jpedsurg.2011.02.036

[2] Valentine, K.M. (2011) Ethanol lock therapy for catheter-associated blood stream infections in a pediatric intensive care unit. Pediatric Critical Care Medicine, 12, e292-e296. http://dx.doi.org/10.1097/PCC.0b013e318219267c

[3] Jones, B.A., Hull, M.A., Richardson, D.S., Zurakowski, D., Gura, K., Fitzgibbons, S.C., et al. (2010) Efficacy of ethanol locks in reducing central venous catheter infections in pediatric patients with intestinal failure. Journal of Pediatric Surgery, 45, 1287-1293. http://dx.doi.org/10.1016/j.jpedsurg.2010.02.099

[4] Wong, T., Clifford, V., McCallum, Z., Shalley, H., Peterkin, M., Paxton, G., et al. (2012) Central venous catheter thrombosis associated with $70 \%$ ethanol locks in pediatric intestinal failure, patients on home parenteral nutrition: A case series. JPEN, 36, 358-360. http://dx.doi.org/10.1177/0148607111414713

[5] Opilla, M.T., Kirby, D.F. and Edmond, M.B. (2007) Use of ethanol lock therapy to reduce the incidence of catheter-related bloodstream infections in home parenteral nutrition patients. JPEN, 31, 302-305. http://dx.doi.org/10.1177/0148607107031004302

[6] Onland, W., Shin, C.E., Fustar, S., Rushing, T. and Wong, W.Y. (2006) Ethanol-lock technique for persistent bacteremia of long-term intravascular devices in pediatric patients. Archives of Pediatrics and Adolescent Medicine, 160, 1049-1053.

http://dx.doi.org/10.1001/archpedi.160.10.1049

[7] Kim, E.Y., Saunders, P. and Yousefzadeh, N. (2010)
Usefulness of anti-infective lock solutions for catheterrelated bloodstream infections. Mount Sinai Journal of Medicine (MSJ), 77, 549-558. http://dx.doi.org/10.1002/msj.20213

[8] Rajpurkar, M., Boldt-MacDonald, K., Mclenon, R., Callaghan, M.U., Chitlur, M., Lusher, J.M., et al. (2009) Ethanol lock therapy for the treatment of catheter-related infections in haemophilia patients. Haemophilia, 15, 12671271.

http://dx.doi.org/10.1111/j.1365-2516.2009.02075.x

[9] Mouw, E., Chessman, K., Lesher, A. and Tagge, A. (2008) Short bowel ethanol. Journal of Pediatric Surgery, 43, 1025-1029.

http://dx.doi.org/10.1016/j.jpedsurg.2008.02.026

[10] Cober, M.P., Kovacevich, D.S. and Teitelbaum, D.H. (2011) Ethanol-lock therapy for the prevention of central venous access device infections in pediatric patients with intestinal failure. JPEN, 35, 67-73. http://dx.doi.org/10.1177/0148607110362758

[11] Metcalf, S.C., Chambers, S.T. and Pithie, A.D. (2009) Use of ethanol locks to prevent recurrent central line sepsis. Journal of Infection, 49, 20-22. http://dx.doi.org/10.1016/j.jinf.2003.08.010

[12] Chambers, S.T., Pithie, A., Gallagher, K., Liu, T., Charles, C.J. and Seaward, L. (2007) Treatment of staphylococcus epidermidis central vascular catheter infection with 70\% ethanol locks: Efficacy in a sheep model. Journal of Antimicrobial Chemotherapy, 59, 779. http://dx.doi.org/10.1093/jac/dkl542

[13] Guenu, S., Heng, A.E., Charbonne, F., et al. (2007) Mass spectrometry and scanning electron microscopy study of silicone tunneled dialysis catheter integrity after an exposure of 15 days to $60 \%$ ethanol solution. Rapid Communications in Mass Spectrometry, 21, 229-236. http://dx.doi.org/10.1002/rcm.2837

[14] Crnich, C.J., Halfmann, J.A., Crone, W.C. and Maki, D.G. (2005) The effects of prolonged ethanol exposure on the mechanical properties of polyurethane and silicone catheters used for intravascular access. Infection Control \& Hospital Epidemiology, 26, 708-714. http://dx.doi.org/10.1086/502607

[15] McGrath, E.J., Salloum, R., Chen, X., Jiang, Y., BoldtMac-Donald, K., Becker, C., et al. (2011) Short-dwell ethanol lock therapy in children is associated with increased clearance of central line-associated bloodstream infections. Clinical Pediatrics, 50, 943-951. http://dx.doi.org/10.1177/0009922811409568

[16] Slobbe, L., Doorduijn, J.K., Lugtenburg, P.J., El Barzouhi, A., Boersma, E., van Leeuwen, W.B., et al. (2010) Prevention of catheter-related bacteremia with a daily ethanol lock in patients with tunnelled catheters: A randomised, placebo-controlled trial. PLOS ONE, 5, e10840. http://dx.doi.org/10.1371/journal.pone.0010840

[17] Bijo, J.K., Maqsood, K.A., Speerhas, R., Rhoda, K., Hamilton, C., DeChicco, R., et al. (2012) Ethanol Lock Therapy in reducing catheter-related bloodstream infections in adult home parenteral nutrition patients: Results of a retrospective study. JPEN, 36, 603-610. http://dx.doi.org/10.1177/0148607111428452 
[18] Dannenberg, C., Bierbach, U., Rothe, A., Beer, J. and Korholz, D. (2003) Ethanol-lock technique in the treatment of bloodstream infections in pediatric oncology patients with broviac catheter. Journal of Pediatric Hematology/Oncology, 25, 616-621.

http://dx.doi.org/10.1097/00043426-200308000-00006

[19] Bisseling, T.M., Willems, M.C., Versleijen, M.W., Hendriks, J.C., Vissers, R.K. and Wanten, G.J. (2010) Taurolidine lock is highly effective in preventing catheter-related bloodstream infectons in patients on home parenteral nutrition: A heparin-controlled prospective trial. Clinical $\mathrm{Nu}$ trition, 29, 464-468. http://dx.doi.org/10.1016/j.clnu.2009.12.005

[20] Torres-Viera, C., Thauvin-Eliopoulos, C., Souli, M., DeGirolami, P., Farris, M.G. and Wennersten, C.B., et al.
(2000) Activities of taurolidine in vitro and in experimental enterococcal endocarditis. Antimicrobial Agents and Chemotherapy, 44, 1720-1724.

http://dx.doi.org/10.1128/AAC.44.6.1720-1724.2000

[21] Dumichen, M.J., Seeger, K., Lode, H.N., Kuhl, J.S., Ebell, W., Degenhardt, P., et al. (2012) Randomized controlled trial of taurolidine citrate versus heparin as catheter lock solution in paediatric patients with haematological malignancies. JHIN, 80, 304-309.

[22] Chu, H.P., Brind, J., Tomar, R. and Hill, S. (2012) Significant reduction in central venous catheter related bloodstream infections in children on home parenteral nutrition after starting treatment with taurolidine line lock. JPGN, 55, 403-407. 